\title{
A fast and comprehensive microdisc laser model applied to all-optical wavelength conversion
}

\author{
J. Hofrichter ${ }^{1}$, O. Raz ${ }^{2}$, F. Horst ${ }^{1}$, N. Chrysos ${ }^{1}$, C. Minkenberg ${ }^{1}$, T. de Vries ${ }^{2}$, H.J.S. Dorren ${ }^{2}$, R. Kumar ${ }^{3}$,
} L. Liu ${ }^{3}$ and B.J. Offrein ${ }^{1}$

1: IBM Research GmbH, IBM Research - Zurich, Säumerstrasse 4, 8803 Rüschlikon, Switzerland 2: COBRA Research Institute, Eindhoven University of Technology, PO. Box 512, 5600MB Eindhoven, The Netherlands

3:Photonics Research Group, INTEC Department, Ghent University - IMEC, St. Pietersnieuwstraat 41, 9000 Ghent, Belgium

Abstract: Microdisc lasers (MDLs) are a viable and attractive option for compact laser sources, wavelength converters and even all-optical optical memory on a chip. Therefore we have developed a fast and comprehensive model for MDLs that is suitable for simulating these functionalities. The simulations are compared with measurements of MDLs operated as alloptical wavelength converters at 10 and $20 \mathrm{~Gb} / \mathrm{s}$.

(C)2010 Optical Society of America

OCIS codes: (000.0000) General; (000.0000) General

\section{Introduction}

Microdisc lasers (MDLs) heterogeneously integrated on silicon wafers [1] as shown in Figs. 1(a) and (b) are an interesting candidate for on-chip laser sources [2], all-optical wavelength converters [3,4] and also all-optical memories [5,6]. In addition, MDLs were used to demonstrate all-optical signal processing and switching [7]. Within a MDL, several modes are competing. Several longitudinal modes may lase simultaneously, originating from the free spectral range (FSR) of the cavity being smaller than the the broad gain spectrum of the MQW gain material. All these modes may lase in both the clockwise and the counter-clockwise direction. Typically several lasing peaks are present as indicated in Fig. 1(c), where the main lasing mode is located at approx. $1585 \mathrm{~nm}$, and the higher wavelength side-mode is present at approx. $1617 \mathrm{~nm}$.
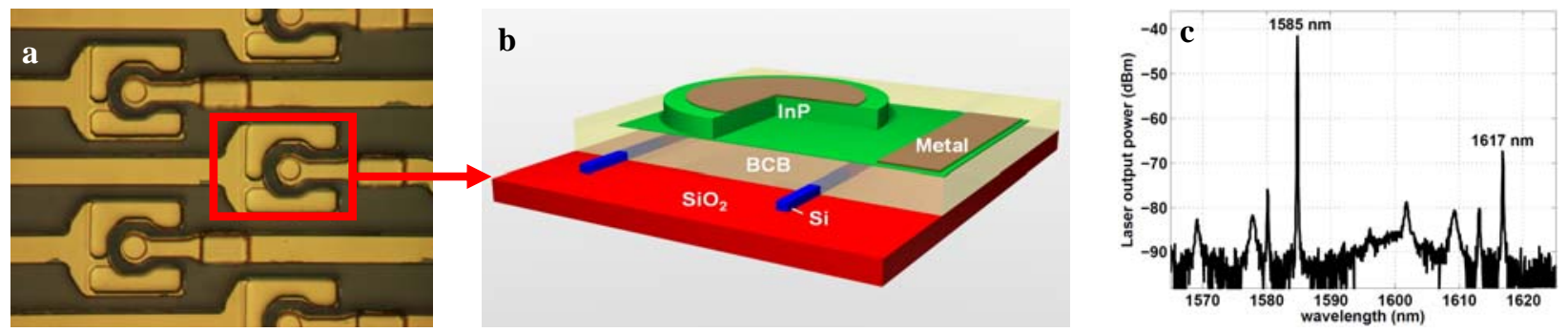

Fig. 1 - A MDL on a SOI waveguide, (a) microscope image, (b) cross section, (c) typical lasing spectrum of a MDL

Earlier numerical models of MDLs have concentrated on the dynamics of two counter propagating modes at the same wavelength $[9,10]$. These models cannot simulate the competition between longitudinal modes in gaindispersive media. There exists a model to describe the latter, using an estimated parabolic dependency of the gain with wavelength [11]. In this work, we have combined these models into a single model, simulating a disc laser having several co- and counter propagating modes. In the next section, the model will be described and in section 3 , simulation results will be compared with measurements [4].

\section{The 1D microdisc laser model}

To accurately simulate the behavior of a MDL as a wavelength converter, we implemented a 1D laser rate equation model. We expanded the model suggested by Van Campenhout et al. [1] for several modes and included self and cross gain suppression with the corresponding factors $\varepsilon_{S}$ and $\varepsilon_{C}$ [8-10]. A parabolic gain curvature was assumed to account for the non-uniform gain spectrum [11]. Equation (1) gives the radially dependent gain term where $G_{0}$ is the maximum gain at the central lasing mode $\lambda_{0}$ and $a$ is the gain curvature parameter. To save computation time and speed up the model, the photon densities $S_{\mathrm{cw}, \mathrm{cc}, \mathrm{i}}$ are modelled as lumped photon densities of the $i$-th mode with the corresponding propagation direction. Because we have a quantum well active material for the given devices, logarithmic gain is assumed, where $N_{0}$ is the threshold carrier density.

$$
g_{C W, C C W}\left(S_{C W}, S_{C C W}, n(r), r\right)=G_{0}\left[1-a\left(\lambda_{0}-\lambda\right)^{2}\right] \cdot\left(1-\varepsilon_{S} \sum_{i} S_{C W, C C W, i}-\varepsilon_{C} \sum_{i} S_{C C W, C W, i}\right) \cdot \ln \frac{n(r)}{N_{0}}
$$

In Eq. (2), the carrier density is locally reduced by stimulated emission into the corresponding mode. Therefore the overlap with the modal distribution $\Psi(\mathrm{r})$ is being calculated. For whispering gallery cavities, $\Psi(\mathrm{r})$ can be approximated by a normalized Bessel function of the first kind $J_{v}\left(r / z_{0}\right)$, where $v$ is the azimuthal order of the 
corresponding mode and $z_{0}$ the first Bessel zero of $J_{v}(z)$. The modal distribution is normalized such that

$$
\begin{aligned}
& \int_{0}^{R} \Psi^{2}(r) r d r=1 . \\
& \frac{\partial n(r)}{\partial t}=-\left[\sum_{i} g_{C W, i}(r) S_{C W, i}+\sum_{i} g_{C C W, i}(r) S_{C C W, i}\right] V_{g} \Psi^{2}(r) \Gamma \pi R^{2}-A n(r)-B n(r)^{2}-C n(r)^{3}+\frac{\eta I}{\pi R^{2} q t_{a}}+D\left(\frac{\partial n(r)}{\partial r}+\frac{\partial^{2} n(r)}{r \partial r^{2}}\right)
\end{aligned}
$$

Besides stimulated emission into the respective lasing modes, also non-lasing recombination is taken into account by the coefficients $A, B$ and $C$ for one, two and three particle processes, respectively. The electrical pumping current $I$ flowing through the disk area $\pi R^{2}$ generates charge carriers in the active region, whose thickness is $t_{\mathrm{a}}$. Regions with depleted carrier concentrations due to stimulated emission in the region of the mode (spatial hole-burning) are refilled by diffusion of charge carriers with the diffusion constant $D$. In contrast to Eqs. (1) and (2) the photon densities defined in Eq. (3) are modelled by lumped variables whose spatial distribution is obtained from $\Psi(r)$. Each photon density experiences a decay time constant $\tau_{\mathrm{p}}$ due to e.g. scattering or absorption.

$$
\begin{aligned}
\frac{\partial S_{C W, C C W, i}}{\partial t}=-\frac{S_{C W, C C W, i}}{\tau_{p}} & +S_{C W, C C W, i} \Gamma v_{g} 2 \pi \int_{0}^{R} g_{C W, C C W, i}(r) \Psi^{2}(r) r \partial r+2 \pi \beta \int_{0}^{R} B n^{2}(r) \Psi^{2}(r) r \partial r \\
& +k_{\mathrm{int}} S_{C C W, C W, i}+k_{e x t} \sqrt{S_{e x t, C W, C C W, i} S_{C W, C C W, i}}
\end{aligned}
$$

The build-up of the mode is achieved by stimulated emission with the modal gain $g_{\mathrm{CW}, \mathrm{CCW}, i}$ in the active region, whose overlap with the mode is $\Gamma$. Also spontaneous emission with the factor $B$ is coupled into the lasing mode with the spontaneous emission coupling factor $\beta$. In addition, scattering due to sidewall roughness causes a coupling of the counter-propagating modes which is modelled by $k_{\text {int }}$ [12]. The external injection coupling factor $k_{\text {ext }}$ models the coherent external injection [9] of light into the respective lasing mode. Table I lists the values of the parameters used in the model. The model's radial dependency enables the simulation of spatial hole-burning which limits output power, modal recovery and thus the device speed.

Table I. Simulation parameters

\begin{tabular}{|c|c|c|c|c|c|c|c|c|c|}
\hline Param. & $R$ & $G_{0}$ & $N_{0}$ & $\varepsilon_{\mathrm{s}}$ & $\varepsilon_{\mathrm{c}}$ & $k_{\text {int }}$ & $k_{\mathrm{ext}}$ & $v_{\mathrm{g}}$ & $\lambda_{0}$ \\
\hline Value & $3.75 \mu \mathrm{m}$ & $1500 \mathrm{~cm}^{-1}$ & $1.5 \mathrm{e} 18 \mathrm{~cm}^{-3}$ & $2 \mathrm{e}-17 \mathrm{~cm}^{3}$ & $4 \mathrm{e}-17 \mathrm{~cm}^{3}$ & $1.5 \mathrm{e} 8 \mathrm{~s}^{-1}$ & $8.8 \mathrm{e} 10 \mathrm{~s}^{-1}$ & $8.817 \mathrm{e} 9 \mathrm{~cm} \mathrm{~s}^{-1}$ & $1550 \mathrm{~nm}$ \\
\hline Param. & $A$ & $B$ & $C$ & $D$ & $\beta$ & $\eta$ & $I$ & $a$ & $t_{\mathrm{a}}$ \\
\hline Value & $1 \mathrm{e}-8 \mathrm{~s}^{-1}$ & $2 \mathrm{e}-10 \mathrm{~cm}^{3} \mathrm{~s}^{-1}$ & $1.63 \mathrm{e}-28 \mathrm{~cm}^{6} \mathrm{~s}^{-1}$ & $8 \mathrm{~cm}^{2} \mathrm{~s}^{-1}$ & $1 \mathrm{e}-3$ & 0.267 & $3 \mathrm{~mA}$ & $1.3 \mathrm{e} 13 \mathrm{~cm}^{-3}$ & $18 \mathrm{~nm}$ \\
\hline
\end{tabular}

\section{Simulations and experiments}

MDLs can be operated as wavelength converters by injecting light into the non-dominant lasing mode, i.e., the mode at approx. $1617 \mathrm{~nm}$ in Fig. 1(c) for our case. Thus, by gain suppression, the previously dominant central lasing mode i.e. the mode at approx. $1586 \mathrm{~nm}$, is suppressed. To operate at high bit rates and high repetition rates, we use the seeding scheme that significantly accelerates the recovery of the central mode by a low-power continuous-wave light injection into the central lasing mode [3,4]. When monitoring the relative eye opening $E O=\left(P_{1, \text { eye }}-P_{0, \text { eye }}\right) / P_{1, \text { max }}$ during wavelength conversion, it becomes clear that seeding improves the eye opening for both $10 \mathrm{~Gb} / \mathrm{s}$ and $20 \mathrm{~Gb} / \mathrm{s}$, As can be seen from Figs. 3(a) and (b), the EO is strongly improved by seeding of continuous-wave light into the central mode.
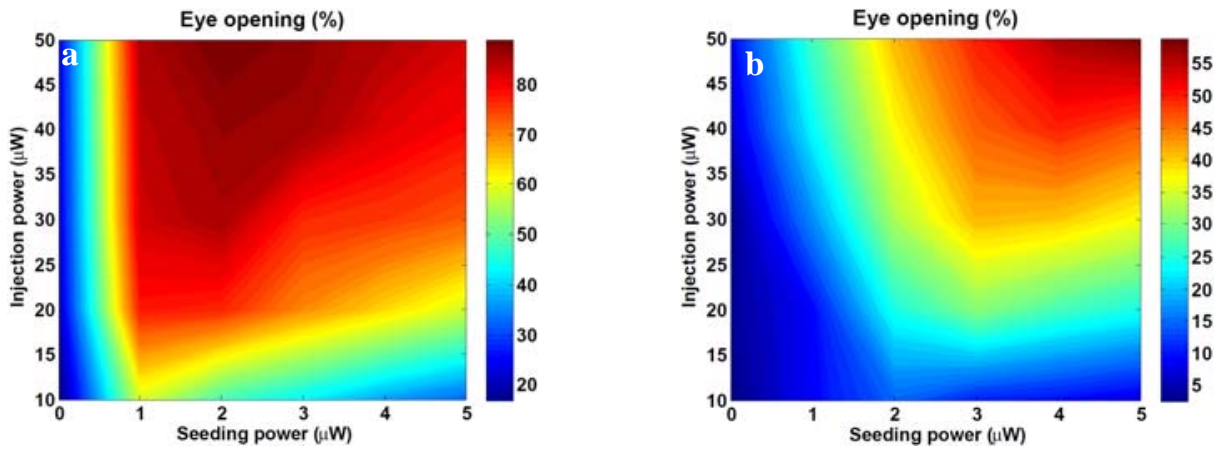

Fig. 3 - Relative eye opening (EO) in \% at (a) $10 \mathrm{~Gb} / \mathrm{s}$ and (b) $20 \mathrm{~Gb} / \mathrm{s}$. 
The eye diagram for $50 \mu \mathrm{W}$ injection in the absence of seeding is reproduced in Figs. 4(a) and (d) for 10 and 20 $\mathrm{Gb} / \mathrm{s}$. Figs. 4(b) and (e) show optimum eye diagrams for the larges EO for 10 and $20 \mathrm{~Gb} / \mathrm{s}$, respectively. The experimental data in Figs. 4(c) and (f) demonstrate excellent agreement with the simulated eye diagrams. Experimentally [4], the scheme of injection seeded disc lasers wavelength converter was shown to operate error-free with a bit-error rate (BER) of below $10^{-9}$ for the operation at $10 \mathrm{~Gb} / \mathrm{s}$ when using seeding. Although the eye for 20 $\mathrm{Gb} / \mathrm{s}$ looks degraded, a BER of below $10^{-3}$ was achieved which is still below the forward-error correction (FEC) limit and thus acceptable for some applications.
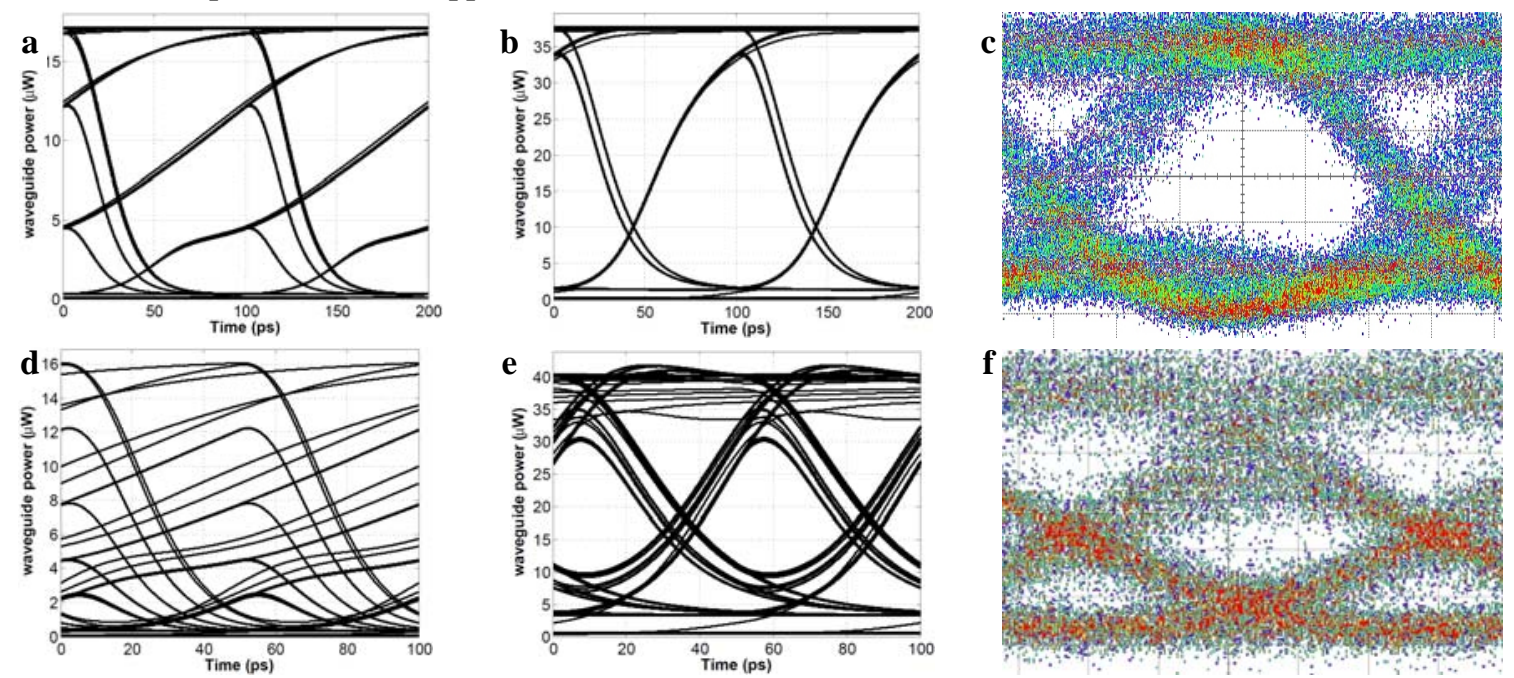

Fig. 4 - Wavelength conversion: Simulation at $10 \mathrm{~Gb} / \mathrm{s}$ with $50 \mu \mathrm{W}$ injection and (a) without seeding, (b) with $1 \mu \mathrm{W}$ seeding, (c) measurement at $10 \mathrm{~Gb} / \mathrm{s}$ with seeding. Simulation at $20 \mathrm{~Gb} / \mathrm{s}$ with $50 \mu \mathrm{W}$ injection and (d) without seeding, (e) with $3 \mu \mathrm{W}$ seeding, (f) measurement at $20 \mathrm{~Gb} / \mathrm{s}$ with seeding.

\section{Conclusion}

We have presented a fast and comprehensive model for the simulation of MDLs. The numerical studies on wavelength conversion of MLDs provided excellent agreement with measurements at both 10 and $20 \mathrm{~Gb} / \mathrm{s}$. It was shown that with seeding, wavelength conversion can be performed at $20 \mathrm{~Gb} / \mathrm{s}$ with an acceptable BER. The model holds promise to facilitate the further exploration of various MDL functionalities and to guide experimental work.

\section{Acknowledgment}

The authors acknowledge the financial support by the European Union FP7-ICT project "HISTORIC".

[1] J. v. Campenhout, P. Rojo-Remeo, P. Regreny, C. Seassal, D. v. Thourhout, S. Verstuyft, L. Di Cioccio, J.-M. Fedeli, C. Lagahe and R. Baets, "Electrically pumped InP-based microdisk lasers integrated with a nanophotonic silicon-on-insulator waveguide circuit,” Opt. Express 15(11), 6744-6749 (2007).

[2] L. Liu, J. v. Campenhout, P. Rojo-Romeo, P. Regreny, C. Seassal, D. v. Thourhout, S. Verstuyft, L. Di Cioccio, J.-M. Fedeli, C. Lagahe and R. Baets, "Compact multiwavelength laser source based on cascaded InP-microdisks coupled to one SOI waveguide," in OSA Technical Digest “Optical Fiber Communication Conf. and Exposition and The National Fiber Optic Engineers Conf.” (OSA, 2008), paper OWQ3.

[3] O. Raz, "High speed wavelength conversion in a heterogeneously integrated disc laser over silicon on insulator for network on a chip applications,” Proc. ECOC, Vienna, Austria, 2009.

[4] O. Raz, "Compact, Low power and low threshold electrically pumped micro disk lasers for 20Gb/s non return to zero all optical wavelength conversion," to be presented at OFC 2010, San Diego, CA, USA, paper OMQ5.

[5] M.T. Hill, “A fast low-power optical memory based on coupled micro-ring lasers,” Nature 432, 206-209 (2004).

[6] L. Liu, R. Kumar, K. Huybrechts, T. Spuesens, G. Roelkens, E.-J. Geluk, T. d. Vries, P. Regreny, D. v. Thourhout, R. Baets and G. Morthier, “An ultra-small, low-power, all-optical flip-flop memory on a silicon chip,” Nature Photonics 268, 1-6 (2010).

[8] W.E. Lamb, Jr., “Theory of an optical maser,” Phys. Rev. A 134, 1429-1450 (1964).

[9] C.L. Tang, A. Schremer and T. Fujita, “Bistability in two-mode semiconductor lasers via gain saturation.” Appl. Phys. Lett. 51(18), 13921394 (1987).

[10] G. Yuan and S. Yu, “Bistability and switching properties of semiconductor ring lasers with external optical injection,” IEEE J. Quantum Electron. 44(1), 41-48, 2008.

[11] M.J. Adams and M. Osihski, “Longitudinal mode competition in semiconductor lasers: Rate equations revisited,” IEE Proc., Part I: Solid State Electron Devices 129(6), 271-274 (1982).

[12] R. Kumar, L. Liu, G. Roelkens, G. Morthier and R. Baets, "Simple and accurate measurements to characterize microdisk lasers for alloptical flip-flop operation,” Proc. Ann. Symp. IEEE Photonics Benelux Chapt., Belgium (2009). 